\title{
Modeling the scattering polarization of the solar Ca I $4227 \AA$ line with angle-dependent partial frequency redistribution
}

\author{
Gioele Janett $^{1,2}$, Ernest Alsina Ballester ${ }^{1,3}$, Nuno Guerreiro ${ }^{1,2}$, Simone Riva ${ }^{1,2}$, Luca Belluzzi $^{1,4,2}$, \\ Tanausú del Pino Alemán ${ }^{3}$, and Javier Trujillo Bueno ${ }^{3,5,6}$
}

\author{
${ }^{1}$ Istituto Ricerche Solari (IRSOL), Università della Svizzera italiana (USI), CH-6605 Locarno-Monti, Switzerland \\ 2 Euler Institute, Università della Svizzera italiana (USI), CH-6900 Lugano, Switzerland \\ 3 Instituto de Astrofísica de Canarias (IAC), E-38205 La Laguna, Tenerife, Spain \\ ${ }^{4}$ Leibniz-Institut für Sonnenphysik (KIS), D-79104 Freiburg i. Br., Germany \\ 5 Departamento de Astrofísica, Universidad de La Laguna, E-38206 La Laguna, Tenerife, Spain \\ ${ }^{6}$ Consejo Superior de Investigaciones Científicas, Spain \\ e-mail: gioele.janett@irsol.usi.ch
}

October 26, 2021

\begin{abstract}
Context. The correct modeling of the scattering polarization signals observed in several strong resonance lines requires taking partial frequency redistribution (PRD) phenomena into account. Modeling scattering polarization with PRD effects is very computationally demanding and the simplifying angle-averaged (AA) approximation is therefore commonly applied.

Aims. This work aims at assessing the impact and the range of validity of the AA approximation with respect to the general angledependent (AD) treatment of PRD effects in the modeling of scattering polarization in strong resonance lines, with focus on the solar Ca I $4227 \AA$ A line.

Methods. Spectral line polarization is modeled by solving the radiative transfer problem for polarized radiation, under nonlocal thermodynamic equilibrium conditions, taking PRD effects into account, in static one-dimensional semi-empirical atmospheric models presenting arbitrary magnetic fields. The problem is solved through a two-step approach. In step 1, the problem is solved for intensity only, considering a multi-level atom. In step 2, the problem is solved including polarization, considering a two-level atom with an unpolarized and infinitely sharp lower level, and fixing the lower level population calculated at step 1.

Results. The results for the Ca I $4227 \AA$ line show a good agreement between the AA and AD calculations for the $Q / I$ and $U / I$ wings signals. However, AA calculations reveal an artificial trough in the line-core peak of the linear polarization profiles, whereas AD calculations show a sharper peak in agreement with observations.

Conclusions. An AD treatment of PRD effects is essential to correctly model the line-core peak of the scattering polarization signal of the Ca I $4227 \AA$ line. By contrast, in the considered static case, the AA approximation seems to be suitable to model the wing scattering polarization lobes and their magnetic sensitivity through magneto-optical effects.
\end{abstract}

Key words. Radiative transfer - Scattering - Polarization - Sun: atmosphere - Methods: numerical

\section{Introduction}

When observing quiet regions close to the edge of the solar disk (limb), several strong resonance lines, such as $\mathrm{H}_{\text {I }} \mathrm{Ly}-\alpha$, Ca II $\mathrm{K}$, Ca I 4227 , or $\mathrm{Na}$ I $\mathrm{D}_{2}$, show significant scattering polarization signals, characterized by a relatively sharp peak in the line core and broader lobes in the wings (e.g., Gandorfer 2000, 2002; Kano et al. 2017). The observation and modeling of these polarization signals has been receiving increasing attention by the scientific community. In fact, their sensitivity to the presence of relatively weak magnetic fields is presently one of the most promising tools to probe the magnetism of the solar chromosphere and transition region (e.g., Trujillo Bueno et al. 2017). This magnetic sensitivity arises from the combined action of the Hanle, Zeeman, and magneto-optical effects (e.g., Alsina Ballester et al. 2017). The interest in these signals is clearly attested by the recent Chromospheric Ly-alpha Spectropolarimeter (CLASP) and Chromospheric Layer SpectroPolarimeter (CLASP2) sounding rocket experiments, which provided unprecedented spectropolarimetric observations of the $\mathrm{H}_{\mathrm{I}}$ Ly-alpha line (Kano et al.
2017; Trujillo Bueno et al. 2018) and of the $\mathrm{Mg}$ II $\mathrm{h}$ and $\mathrm{k}$ lines (Ishikawa et al. 2021), respectively. In the visible and infrared ranges of the solar spectrum, scattering polarization signals of strong chromospheric lines can be observed from the ground with instruments such as the Zurich Imaging Polarimeter (ZIMPOL, Ramelli et al. 2010) or the Tenerife Infrared Polarimeter II (TIP-II, Collados et al. 2007), and will be among the targets of the new generation of large-aperture solar telescopes (e.g., DKIST and EST).

The scattering polarization signals of strong resonance lines are primarily produced by scattering processes that are coherent in frequency in the atomic reference frame, and subject to Doppler redistribution in the observer's frame. The core of these signals can be often suitably modeled under the limit of complete frequency redistribution (CRD; e.g., Sampoorna et al. 2010; Štěpán 2015). However, this approximation turns out to be completely inadequate to model their extended wing lobes, for which a general approach accounting for partial frequency redistribution (PRD) effects is of the utmost importance. The numerical solution of the radiative transfer (RT) problem for 
polarized radiation under nonlocal thermodynamic equilibrium (NLTE) conditions, taking scattering polarization and PRD effects into account is a notoriously challenging problem from the computational point of view. For this reason, the so-called angle-averaged (AA) simplifying assumption, first introduced in the unpolarized case (e.g., Mihalas 1978), is still commonly applied to the modeling of scattering polarization signals (see, e.g., Rees \& Saliba 1982; Belluzzi \& Trujillo Bueno 2014; Alsina Ballester et al. 2017). By smearing out geometrical aspects of the problem, the AA assumption can, however, introduce significant inaccuracies, and it would be completely inadequate when aiming at modeling the aforementioned signals in realistic atmospheric models, which consider the detailed threedimensional (3D) structure of the solar atmosphere.

This highlights the need for methods that, exploiting the increased computational resources available today, make it possible to model these signals by solving the NLTE RT problem for polarized radiation, taking PRD effects into account in the general angle-dependent (AD) case. In fact, considerable efforts have already been put in this direction. Dumont et al. (1977) pioneered the modeling of spectral line polarization accounting for AD PRD effects. Subsequently, Nagendra et al. (2002) considered the AD PRD problem in the weak-field Hanle regime for the case of a two-level atom, using the theoretical approach of Stenflo (1994), based on the Kramers-Heisenberg formula. Sampoorna et al. (2008, 2017) generalized that work to the Hanle-Zeeman regime considering arbitrary magnetic fields. Sampoorna et al. (2011) and Supriya et al. (2012) discussed the validity of the AA assumption in the presence of micro-turbulent magnetic fields, while Supriya et al. (2013) extended the analysis to vertical magnetic fields. Sampoorna \& Nagendra (2015) developed an AD PRD RT code capable of including vertical bulk velocities. For computational simplicity, the AD PRD calculations presented in the aforementioned works were performed considering one-dimensional (1D) homogeneous atmospheric models. More recently, del Pino Alemán et al. (2020) solved the AD PRD problem for a three-term atomic model in more realistic 1D atmospheric models, applying the theoretical approach of Casini et al. (2014, 2017). Their code can take into account the effect of AD PRD in the presence of magnetic fields of arbitrary strength and orientation, as well as vertical bulk velocities. However, their AD PRD calculations were limited to problems with axial symmetry, because of the extreme computational cost.

This paper shows the results obtained with a new code capable of solving the NLTE RT problem in static 1D semi-empirical models of the solar atmosphere, taking scattering polarization and AD PRD effects into account. Based on the theoretical approach developed by Bommier (1997a,b), the code allows considering two-level atomic models with an unpolarized and infinitely sharp lower level, in the presence of arbitrary magnetic fields. The present investigation is specifically focused on the Ca I $4227 \AA$ line, which is an ideal test bench for new approaches to the modeling of scattering polarization including PRD effects. Indeed, the scattering polarization signal of this line clearly shows the impact of such effects (Faurobert-Scholl 1992) and can be suitably modeled by considering a simple twolevel atom. Furthermore, the Stokes profiles of this line are of high scientific interest for magnetic field diagnostics, and highprecision spectropolarimetric observations are available (e.g., Bianda et al. 2011; Supriya et al. 2014; Alsina Ballester et al. 2018; Capozzi et al. 2020).

The logical structure of this paper is as follows: Section 2 outlines the formulation of the problem, exposing the relevant continuous equations and their discretization, and presenting a suitable iterative numerical approach to solve it. Next, Section 3 focuses on the modeling of the Ca I $4227 \AA$ line, presenting a quantitative comparison between the results of AA and AD calculations. Finally, Section 4 summarizes the main results of this paper and discusses on their implications.

\section{Formulation of the problem and methods}

In this section, we first present the continuous formulation of the considered NLTE RT problem for polarized radiation, including AD PRD effects. We then expose the discretization and the algebraic formulation of the problem and outline the numerical iterative solution method that was applied. In addition, we finally recall the simplifying AA approximation.

\subsection{Continuous formulation of the problem}

The physical quantities entering the problem are, in general, functions of the spatial point $\mathbf{r}$, and of the frequency $v$ and propagation direction $\boldsymbol{\Omega}$ of the radiation beam under consideration. A complete description of the polarization state of the radiation field is provided by the four Stokes parameters

$$
I_{i}(\mathbf{r}, \boldsymbol{\Omega}, v)
$$

with $i=1, \ldots, 4$, standing for Stokes $I, Q, U$, and $V$, respectively. The transfer of partially polarized light along direction $\boldsymbol{\Omega}$ at frequency $v$ is described by the system of coupled first-order inhomogeneous ordinary differential equations given by

$$
\frac{\mathrm{d}}{\mathrm{d} s} I_{i}(\mathbf{r}, \boldsymbol{\Omega}, v)=-\sum_{j=1}^{4} K_{i j}(\mathbf{r}, \mathbf{\Omega}, v) I_{j}(\mathbf{r}, \mathbf{\Omega}, v)+\varepsilon_{i}(\mathbf{r}, \mathbf{\Omega}, v),
$$

where $s$ is the spatial coordinate measured along the direction $\boldsymbol{\Omega}$, $K_{i j}$ are the elements of the so-called propagation matrix

$$
K=\left(\begin{array}{cccc}
\eta_{1} & \eta_{2} & \eta_{3} & \eta_{4} \\
\eta_{2} & \eta_{1} & \rho_{4} & -\rho_{3} \\
\eta_{3} & -\rho_{4} & \eta_{1} & \rho_{2} \\
\eta_{4} & \rho_{3} & -\rho_{2} & \eta_{1}
\end{array}\right),
$$

where $\eta_{i}$ and $\rho_{i}$ are the dichroism coefficients and the anomalous dispersion coefficients, respectively, while $\varepsilon_{i}$ are the emission coefficients in the four Stokes parameters. In general, $K_{i j}$ and $\varepsilon_{i}$ contain contributions due to both line and continuum processes and can be expressed as

$$
\begin{aligned}
K_{i j}(\boldsymbol{r}, \boldsymbol{\Omega}, v) & =K_{i j}^{\ell}(\boldsymbol{r}, \boldsymbol{\Omega}, v)+K_{i j}^{c}(\boldsymbol{r}, v), \\
\varepsilon_{i}(\boldsymbol{r}, \boldsymbol{\Omega}, v) & =\varepsilon_{i}^{\ell}(\boldsymbol{r}, \boldsymbol{\Omega}, v)+\varepsilon_{i}^{c}(\boldsymbol{r}, \boldsymbol{\Omega}, v),
\end{aligned}
$$

with the superscripts $\ell$ and $c$ standing for line and continuum, respectively.

In this work, we consider a two-level atomic model with an unpolarized and infinitely sharp lower level in the presence of magnetic fields of arbitrary intensity and orientation. For such an atomic model, an analytical solution of the statistical equilibrium equations is available and the line contribution to the emission coefficient can be thus expressed through the redistribution matrix formalism. Stimulated emission is negligible around the Ca I $4227 \AA$ frequency in the solar atmosphere and is consequently neglected. Moreover, bulk velocity fields are not considered; their impact on this problem will be the subject of an upcoming investigation. 


\subsubsection{Line contributions}

The line contributions to $\eta_{i}$ and $\rho_{i}$ for the considered atomic model are given by (see Alsina Ballester et al. 2017)

$\eta_{i}^{\ell}(\mathbf{r}, \boldsymbol{\Omega}, v)=k_{L}(\mathbf{r}) \sum_{K=0}^{2} \hat{\mathcal{T}}_{0, i}^{K}(\mathbf{r}, \boldsymbol{\Omega}) \Phi_{0}^{0 K}(\mathbf{r}, v)$,

$\rho_{i}^{\ell}(\mathbf{r}, \boldsymbol{\Omega}, v)=k_{L}(\mathbf{r}) \sum_{K=0}^{2} \hat{\mathcal{T}}_{0, i}^{K}(\mathbf{r}, \mathbf{\Omega}) \Psi_{0}^{0 K}(\mathbf{r}, v)$,

where $k_{L}$ is the frequency-integrated absorption coefficient, which depends on the population of the lower level, $\hat{\mathcal{T}}_{Q, i}^{K}$ is the polarization tensor (see Landi Degl'Innocenti \& Landolfi 2004, Chapter 5) evaluated in the magnetic reference system ${ }^{1}$, while $\Phi_{Q}^{K K^{\prime}}$ and $\Psi_{Q}^{K K^{\prime}}$ are the generalized profiles defined in Landi Degl'Innocenti \& Landolfi (2004, Appendix 13).

Within the redistribution matrix formalism, one can distinguish between thermal and scattering contributions to the line emission coefficients, namely,

$\varepsilon_{i}^{\ell}(\mathbf{r}, \boldsymbol{\Omega}, v)=\varepsilon_{i}^{\ell, t h}(\mathbf{r}, \boldsymbol{\Omega}, v)+\varepsilon_{i}^{\ell, s c}(\mathbf{r}, \boldsymbol{\Omega}, v)$.

Assuming that inelastic collisions are isotropic, the thermal contribution to the line emission coefficients for the considered atomic system reads (see Alsina Ballester et al. 2017)

$$
\begin{aligned}
\varepsilon_{i}^{\ell, t h}(\mathbf{r}, \boldsymbol{\Omega}, v) & =k_{L}(\mathbf{r}) \epsilon(\mathbf{r}) W_{T}(\mathbf{r}) \sum_{K=0}^{2} \hat{\mathcal{T}}_{0, i}^{K}(\mathbf{\Omega}) \Phi_{0}^{0 K}(\mathbf{r}, v) \\
& =\epsilon(\mathbf{r}) W_{T}(\mathbf{r}) \eta_{i}^{\ell}(\mathbf{r}, \mathbf{\Omega}, v),
\end{aligned}
$$

where $\epsilon$ is the thermalization parameter, and $W_{T}$ is the Planck function in the Wien limit at the line-center frequency $v_{0}$. The scattering contribution to the line emission coefficients reads

$\varepsilon_{i}^{\ell, s c}(\mathbf{r}, \boldsymbol{\Omega}, v)=k_{L}(\mathbf{r}) \int \mathrm{d} v^{\prime} \oint \frac{\mathrm{d} \boldsymbol{\Omega}^{\prime}}{4 \pi} \sum_{j=1}^{4} R_{i j}\left(\mathbf{r}, \boldsymbol{\Omega}, \boldsymbol{\Omega}^{\prime}, v, v^{\prime}\right) I_{j}\left(v^{\prime}, \boldsymbol{\Omega}^{\prime}, \mathbf{r}\right)$,

where $R_{i j}$ are the elements of the redistribution matrix and $I_{j}$ are the Stokes parameters of the radiation that pumps the atomic system. Hereafter, the primed and unprimed quantities refer to the incoming and outgoing radiation, respectively. The redistribution matrix derived by Bommier (1997b) is given by the sum of two terms

$R_{i j}\left(\mathbf{r}, \boldsymbol{\Omega}, \mathbf{\Omega}^{\prime}, v, v^{\prime}\right)=R_{i j}^{\mathrm{II}}\left(\mathbf{r}, \boldsymbol{\Omega}, \mathbf{\Omega}^{\prime}, v, v^{\prime}\right)+R_{i j}^{\mathrm{III}}\left(\mathbf{r}, \mathbf{\Omega}, \mathbf{\Omega}^{\prime}, v, v^{\prime}\right)$,

where $R^{\mathrm{II}}$ and $R^{\mathrm{III}}$ describe scattering processes that are coherent and completely uncoherent, respectively, in the atomic reference frame. The transformation of $R^{\mathrm{II}}$ of Bommier (1997b) from the atomic to the observer's frame (accounting for the Doppler effect due to thermal motions) is described in detail in Alsina Ballester et al. (2017). The assumption of CRD in the observer's frame is instead made for $R^{\mathrm{III}}$ (e.g., Bommier 1997b; Alsina Ballester et al. 2017). This approximation appears to be suitable for modeling strong chromospheric lines, whose core forms at atmospheric heights where the rate of elastic collisions is relatively low, and the contribution of $R^{\mathrm{III}}$ is therefore much smaller than that of $R^{\mathrm{II}}$. A quantitative analysis of the impact of this approximation on our modeling goes beyond the scope

\footnotetext{
${ }^{1}$ It is the reference system with the $z$-axis directed along the local magnetic field vector, which depends on the spatial point $\mathbf{r}$. This explains the dependence of the polarization tensor $\hat{\mathcal{T}}_{Q, i}^{K}$ on $\mathbf{r}$.
}

of this work and is left for a future investigation. The reader is referred to Bommier (1997b) and Sampoorna et al. (2017) for more details on the suitability of this assumption. The ensuing redistribution matrices can be written as

$$
\begin{aligned}
& R_{i j}^{\mathrm{II}}\left(\mathbf{r}, \boldsymbol{\Omega}, \boldsymbol{\Omega}^{\prime}, v, v^{\prime}\right)=\sum_{K K^{\prime} Q} \mathcal{R}_{Q}^{\mathrm{II}, K K^{\prime}}\left(\mathbf{r}, v, v^{\prime}, \Theta\right) P_{Q, i j}^{K K^{\prime}}\left(\boldsymbol{\Omega}, \mathbf{\Omega}^{\prime}\right), \\
& R_{i j}^{\mathrm{II}}\left(\mathbf{r}, \boldsymbol{\Omega}, \boldsymbol{\Omega}^{\prime}, v, v^{\prime}\right)=\sum_{K K^{\prime} Q} \mathcal{R}_{Q}^{\mathrm{III}, K K^{\prime}}\left(\mathbf{r}, v, v^{\prime}\right) P_{Q, i j}^{K K^{\prime}}\left(\boldsymbol{\Omega}, \boldsymbol{\Omega}^{\prime}\right),
\end{aligned}
$$

where $\Theta$ represents the scattering angle, that is the angle between the incoming $\boldsymbol{\Omega}^{\prime}$ and outgoing $\boldsymbol{\Omega}$ directions. The redistribution functions $\mathcal{R}_{Q}^{\mathrm{II}, K K^{\prime}}$ and $\mathcal{R}_{Q}^{\mathrm{II}, K K^{\prime}}$ and the elements of the scattering phase matrix $P_{Q, i j}^{K K^{\prime}}$ are also described in Alsina Ballester et al. (2017).

\subsubsection{Continuum contributions}

In the visible part of the solar spectrum, continuum processes only contribute significantly to the diagonal element $\eta_{I}$ of the propagation matrix, namely,

$$
\begin{aligned}
\eta_{i}^{c}(\mathbf{r}, v) & =k_{c}(\mathbf{r}, v) \delta_{i 1}, \\
\rho_{i}^{c} & =0,
\end{aligned}
$$

where $\delta_{i j}$ is the Kronecker delta. The continuum contribution to the emission coefficients can be divided into a (unpolarized and isotropic) thermal term and a scattering term

$\varepsilon_{i}^{c}(\mathbf{r}, \boldsymbol{\Omega}, v)=\varepsilon_{I}^{c, t h}(\mathbf{r}, v) \delta_{i 1}+\varepsilon_{i}^{c, s c}(\mathbf{r}, \boldsymbol{\Omega}, v)$.

The scattering contribution to the continuum emission coefficients reads (see Alsina Ballester et al. 2017)

$$
\varepsilon_{i}^{c, s c}(\mathbf{r}, \boldsymbol{\Omega}, v)=\sigma(\mathbf{r}, v) \sum_{K Q}(-1)^{Q} \mathcal{T}_{Q, i}^{K}(\mathbf{\Omega}) \oint \frac{\mathrm{d} \boldsymbol{\Omega}^{\prime}}{4 \pi} \sum_{j=1}^{4} \mathcal{T}_{-Q, j}^{K}(\boldsymbol{\Omega}) I_{j}(\mathbf{r}, \boldsymbol{\Omega}, v),
$$

where $\sigma$ is the continuum opacity for scattering and $\mathcal{T}_{Q, i}^{K}$ is the polarization tensor (see Landi Degl'Innocenti \& Landolfi 2004, Chapter 5) evaluated in the reference system of the problem ${ }^{2}$.

In summary, in order to determine the Stokes parameters $I_{i}$ of the radiation emerging from the considered atmospheric model, one needs to solve the transfer equation (1). This requires the knowledge of the emission vector $\varepsilon_{i}$ which, in turn, depends on the Stokes parameters $I_{i}$ through (7) and (9). The problem thus consists in finding a self-consistent solution for $I_{i}$ and $\varepsilon_{i}$.

\subsection{Discretization and algebraic formulation}

After a suitable discretization of the continuous problem (see, e.g., Janett et al. 2021), the solution of the transfer equation (1) and the calculation of the emission coefficients (3) can be expressed in the compact matrix form

$$
\begin{array}{ll}
\mathbf{I}=\Lambda \boldsymbol{\varepsilon}+\mathbf{t}, & \text { with } \Lambda \in \mathbb{R}^{4 N_{s} N_{\Omega} N_{v} \times 4 N_{s} N_{\Omega} N_{v}}, \\
\boldsymbol{\varepsilon}=\Sigma \mathbf{I}+\mathbf{c}, & \text { with } \Sigma \in \mathbb{R}^{4 N_{s} N_{\Omega} N_{v} \times 4 N_{s} N_{\Omega} N_{v}},
\end{array}
$$

respectively, with the vectors $\mathbf{I}, \boldsymbol{\varepsilon}, \mathbf{t}, \mathbf{c} \in \mathbb{R}^{4 N_{s} N_{\Omega} N_{v}}$. I collects the discretized Stokes parameters and $\boldsymbol{\varepsilon}$ the discretized emission coefficients. Moreover, $\Lambda \boldsymbol{\varepsilon}$ describe the transfer of the radiation

\footnotetext{
2 It is the reference system with the $z$-axis directed along the vertical of the atmospheric model.
} 
generated inside the atmospheric model, $\mathbf{t}$ represents the radiation transmitted from the boundaries, while $\Sigma \mathbf{I}$ and $\mathbf{c}$ represent the scattering and thermal contributions to the emission coefficients, respectively.

By choosing I as the problem's unknown, Equations (10)(11) can then be combined in a single discrete problem, namely,

$(I d-\Lambda \Sigma) \mathbf{I}=\Lambda \mathbf{c}+\mathbf{t}$,

where $I d$ is the identity matrix of size $4 N_{s} N_{\Omega} N_{v}$. The discrete problem (12) is generally nonlinear, because the coefficients of $\Lambda$ and $\mathbf{t}$ depend in a nonlinear way on the absorption coefficient $\eta_{I}$, which depends on the lower level atomic population, which in turn is affected by the unknown I. However, if the lower level atomic population is fixed a priori, the absorption coefficient $\eta_{I}$ becomes a constant of the problem. In this case, $\Lambda$ and $\mathbf{t}$ become independent from the unknown I and the discrete problem (12) becomes linear.

\subsection{Numerical iterative solution}

The numerical solution of the full NLTE RT problem is divided into two main steps:

1. The nonlinear NLTE RT problem is first solved considering a multi-level atomic model, taking PRD effects into account, but neglecting polarization and the effect of magnetic fields. An accurate estimate of the populations of the atomic levels is thus provided.

2. A two-level atomic model with an unpolarized and infinitely sharp lower level is considered. The population of the lower level obtained in the first step is used and kept fixed. The resulting linear problem (12) is solved in the general AD case. Although this problem can in principle be solved in a single step with a direct method, this task is beyond current computational capabilities. A stationary or Krylov iterative method can therefore be applied (see, e.g., Janett et al. 2021; Benedusi et al. 2021), using the unpolarized radiation field obtained in the first step as an initial guess.

Although it is not fully consistent, this two-step approach permits to obtain an accurate estimate of the lower level population from a multilevel atomic model, while retaining the simplicity of a two-level atom when accounting for polarization. Moreover, by fixing the lower level population, the line absorption coefficient becomes a constant, and the problem at step 2 becomes linear (see Section 2.2). It is worth observing that the population of the upper level is not kept fixed at step 2. This allows taking into account the impact of atomic polarization (neglected at step 1) on the ratio between the population of the upper and lower level. In this respect, it must be observed that the degree of atomic polarization is in general quite low, and therefore this impact is in most cases very small. The validity of the approach is corroborated by previous works (e.g., Belluzzi \& Trujillo Bueno 2012; Belluzzi et al. 2012; Alsina Ballester et al. 2016, 2018).

\subsection{Angle-averaged approximation}

In the general $\mathrm{AD}$ approach, one considers the inherent coupling between incoming and outgoing directions and frequencies, which manifests in presence of the scattering angle $\Theta$ in the redistribution function $\mathcal{R}_{Q}^{\mathrm{II}, K K^{\prime}}$. Accurate $\mathrm{AD}$ calculations are computationally very expensive, and the AA approximation (e.g., Mihalas 1978; Rees \& Saliba 1982) was thus proposed to simplify the numerical problem. According to the formulation outlined in Sect. 2.1, this approximation consists in averaging the expression of $\mathcal{R}_{Q}^{\mathrm{II}, K K^{\prime}}$ over the scattering angle $\Theta$, namely,

$\overline{\mathcal{R}}_{Q}^{\mathrm{II}, K K^{\prime}}\left(\mathbf{r}, v, v^{\prime}\right)=\frac{1}{2} \int_{0}^{\pi} \mathrm{d} \Theta \sin (\Theta) \mathcal{R}_{Q}^{\mathrm{II}, K K^{\prime}}\left(\mathbf{r}, \Theta, v, v^{\prime}\right)$.

The AA redistribution function $\overline{\mathcal{R}}_{Q}^{\mathrm{II}, K K^{\prime}}$ is thus independent of the scattering angle, partially decoupling (in the absence of bulk velocities) frequencies and directions in the calculation of the line emissivity (see Equation (7)). This allows for a drastic reduction of the computational cost (e.g., Alsina Ballester et al. 2017).

This approximation proved suitable to model the intensity profiles (e.g., Leenaarts et al. 2012; Sukhorukov \& Leenaarts 2017). In the modeling of polarization signals, the range of validity of this approximation was analyzed by Faurobert (1987, 1988) for the nonmagnetic case, by Sampoorna et al. (2011) for weak magnetic fields, and by Sampoorna et al. (2017) for arbitrary magnetic fields. The overall conclusion is that the AA approximation does not allow a satisfactory modeling of the linear polarization signals in the core of spectral lines. These analyses have been, however, limited to academic scenarios, with hypothetical lines and homogeneous atmospheric models. A rigorous comparison between the AA and the AD approaches for realistic atomic and atmospheric models is still lacking and is the main scope of the next section.

\section{Numerical results}

This section presents the numerical modeling of the linear scattering polarization profiles of the Ca I line at $4227 \AA$, as an illustrative application of the problem's formulation, discretization, and solution method described in Section 2. The calculations are carried out in 1D semi-empirical models of the solar atmosphere, in the presence of arbitrary magnetic fields, and taking AD PRD effects into account.

\subsection{The Ca I $4227 \AA$ spectral line}

The Ca I $4227 \AA$ line is produced by the transition between the ground level of neutral calcium $\left(4 s^{2}{ }^{1} \mathrm{~S}_{0}\right)$ and the excited level $4 s 4 p^{1} \mathrm{P}_{1}^{\mathrm{o}}$. Since both levels pertain to singlets and the upper one is not radiatively connected to other levels with lower energy, the scattering polarization signal of this line can be suitably modeled by considering a two-level atomic model.

The intensity spectrum of this line shows extended damping wings and a nearly saturated line core. For a disk center line of sight (LOS) and considering the semi-empirical model $\mathrm{C}$ of Fontenla et al. (1993), the line core forms at a height of about $900 \mathrm{~km}$ above the $\tau=1$ surface, with $\tau$ the continuum optical depth at $5000 \AA$ along the vertical. When observed in quiet regions close to the limb, this line exhibits the largest scattering polarization signal in the visible range of the so-called Second Solar Spectrum (e.g., Stenflo et al. 1980; Stenflo 1982; Gandorfer 2002). This signal presents a clear triple-peak structure, typical of strong resonance lines, with a sharp peak in the line core and broad lobes in the wings. The wing lobes form at photospheric heights (at around $180 \mathrm{~km}$ for an observation at $\mu=\cos \theta=0.1$, with $\theta$ the heliocentric angle), and can only be suitably modeled by taking PRD effects into account. The scattering polarization signal of this line is of particular interest because of its sensitivity to the magnetic fields present across a wide range of atmospheric heights. Indeed, the line-core peak is sensitive to low-chromospheric magnetic fields through the Hanle effect 
(Stenflo 1982; Faurobert-Scholl 1992), while the wing lobes are sensitive to photospheric fields via magneto-optical effects (Alsina Ballester et al. 2018). Furthermore, the well-known Zeeman effect can be exploited as well in this line.

A considerable effort was indeed directed to the observation and modeling of the scattering polarization signal of the $\mathrm{Ca}$ I $4227 \AA$ line. Worth of mention are the observations of Bianda et al. (2003), which showed unexpected $U / I$ wing signals, as well as clear spatial variations of the amplitude of both the $Q / I$ and $U / I$ wing lobes. These observations could be explained in terms of magneto-optical effects by Alsina Ballester et al. (2018). This theoretical interpretation has gained further support from the observational work by Capozzi et al. (2020). In the core of this line, scattering polarization signals can also be observed at the disk center, the interpretation of which requires $3 \mathrm{D}$ radiative transfer modeling (see Jaume Bestard et al. 2021). Observations of such diskcenter signals were presented by Bianda et al. (2011), and subsequently analyzed by Anusha et al. (2011) to infer the magnetic fields present in the low chromosphere under the assumption that the observed signals are due to the Hanle effect in forward-scattering geometry (see Trujillo Bueno 2001). More recently, Carlin \& Bianda (2017) theoretically studied the temporal evolution of these polarization signals using the CRD and $1.5 \mathrm{D}$ approximations, emphasizing their sensitivity to gradients in the vertical component of the bulk velocities, while Jaume Bestard et al. (2021) investigated the linear and circular polarization signals of the Ca I $4227 \AA$ line in full 3D, pointing out the important symmetry breaking produced by the spatial gradients of the horizontal component of the macroscopic velocities. Moreover, Supriya et al. (2014) modeled the centerto-limb variation of the $Q / I$ profile of this line, and compared it to observations recorded with the ZIMPOL-III instrument (e.g., Ramelli et al. 2010).

\subsection{Numerical setting and methods}

The Stokes profiles of the Ca I $4227 \AA$ line presented in the following sections were calculated in the 1D semi-empirical models of Fontenla et al. (1993, hereafter FAL models), whose spatial grids contain between 60 and 70 nodes. A Cartesian reference system with the $z$-axis (quantization axis for total angular momentum) directed along the vertical is considered. A given propagation direction $\boldsymbol{\Omega}$ is specified through its inclination $\theta \in[0, \pi]$ (i.e., $\mu=\cos \theta \in[-1,1]$ ) with respect to the vertical, and azimuth $\chi \in[0,2 \pi)$ measured counter-clockwise from the $x$-axis. The angular grid is given by the tensor product of 8 equally spaced nodes in the azimuthal interval $[0,2 \pi]$, and 6 Gauss-Legendre quadrature nodes in each inclination subinterval $[-1,0]$ and $[0,1]$, for a total of 96 nodes. The wavelength interval [ $4071 \AA, 4261 \AA]$ is discretized onto a spectral grid with 155 nodes, equally spaced in the line core and logarithmically distributed in the wings. In all calculations, the reference direction for positive Stokes $Q$ is taken parallel to the limb.

The whole problem is solved through the two-step approach presented in Section 2.3. The first step is carried out by means of the RH code (Uitenbroek 2001), using the parabolic shortcharacteristic formal solver. In step 1 , an atomic model for calcium with 25 levels ( $19 \mathrm{Ca}$ I levels, 5 Ca II levels, and the ground level of $\mathrm{Ca}$ III) is considered. This model accounts for 21 line transitions and 24 continuum transitions. All spectral lines are computed in the CRD limit, except for the Ca I $4227 \AA$ line and the $\mathrm{Ca}$ II $\mathrm{H} \& \mathrm{~K}$ lines, which receive a PRD treatment. The $\mathrm{RH}$ code is also used to calculate the continuum total opacity $k_{c}$, scattering opacity $\sigma$, and thermal emissivity $\varepsilon_{I}^{c, t h}$, as well as the collisional rates. The rates for inelastic collisions with electrons, inducing transitions between the upper and lower levels of the Ca I $4227 \AA$ line, are computed according to the formula given in Seaton (1962). The rates of elastic collisions are calculated including the contributions from the Van der Waals interaction with neutral hydrogen and helium atoms, computed following Unsöld (1955), and from the quadratic Stark effect, due to electrons and singly charged ions (Traving 1960; Gray 1992).

Step 2 is carried out by means of a Matlab code that solves the linear problem (12) in the general AD case with a Richardson iterative method (see Janett et al. 2021), using the unpolarized radiation field calculated at step 1 as initial guess. In step 2 , a two-level atomic model is considered. The population of the lower level is fixed to the value calculated in the first step and left unchanged. The DELO-parabolic formal solver (Janett et al. 2017,2018 ) is used to solve the transfer equation (1).

Concerning the choice of the formal solver, it must be recalled that high-order schemes generally outperform low-order methods if the atmospheric model guarantees sufficient smoothness in the solution. This turns out to be the case for the FAL models, and for this reason, the third-order accurate DELOparabolic method of Janett et al. (2017) was used to perform the calculations presented in this paper. Even though Janett et al. (2018) did not recommend multistep schemes for practical applications, the smoothness of the FAL atmospheric models allows the safe use of the DELO-parabolic method, guaranteeing its proximity to $L$-stability (see Janett \& Paganini 2018).

In order to facilitate the convergence in step 2, an intermediate step can be added. In this additional step, the linear problem (12) is solved with a stationary iterative method under the AA approximation (see Alsina Ballester et al. 2017). This calculation provides temporary solution for the polarized radiation field, which acts as a better initial guess for the step 2. At the same time, the Stokes profiles resulting from the intermediate step also provides a comparison suitable to identify the impact and the range of validity of the AA approximation with respect to the general AD treatment of PRD effects. These AA calculations are carried out using a modified version of the Fortran code developed by Alsina Ballester et al. (2017), which solves the linear problem (12) taking PRD effects into account under the AA approximation. Unlike the original version of the code, this modified version uses a damped Jacobi iterative method ${ }^{3}$ and the DELO-parabolic formal solver. The damped Jacobi method is used because the Jacobi iterative method does not guarantee convergence when used in combination with the DELOparabolic formal solver (see Janett et al. 2021). A detailed analysis on the convergence properties of various iterative methods applied to transfer problems of polarized radiation can be found in Janett et al. (2021).

\subsection{Nonmagnetic case}

The impact of the AD treatment on the modeling of the emergent Stokes profiles of the Ca I $4227 \AA$ line is first analyzed in the absence of magnetic fields, considering the four different FAL models. These atmospheric models represent a faint internetwork region of the quiet Sun (FAL-A), an average region of

\footnotetext{
3 The idea of damping consists in multiplying the correction in the Jacobi iteration by a damping parameter (or relaxation factor) $\omega$, which typically satisfies $0<\omega<1$ (see, e.g., Hackbusch 1994; Janett et al. 2021).
} 


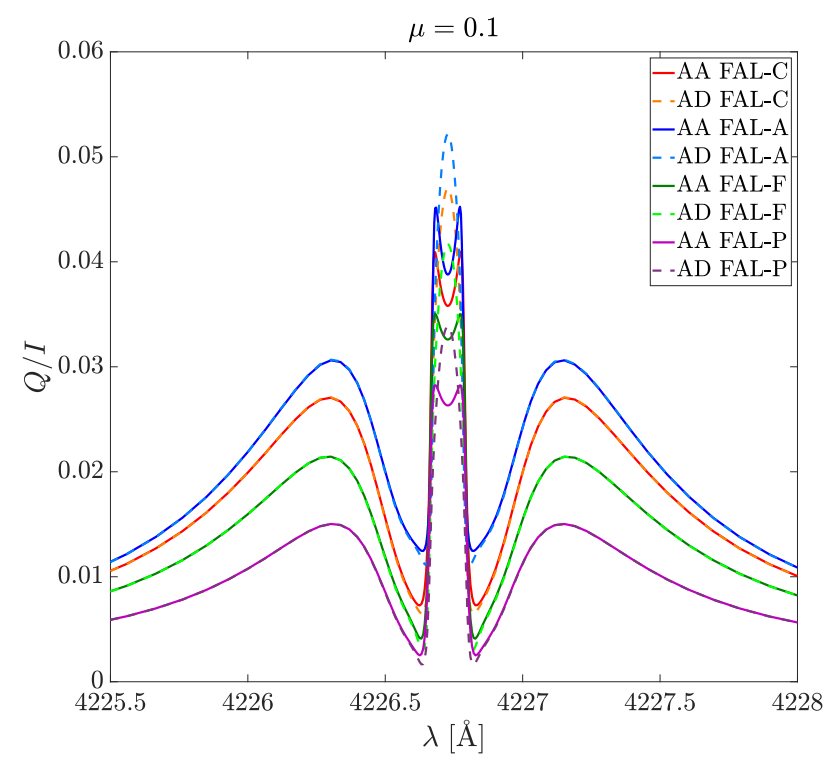

Fig. 1. Emergent $Q / I$ profiles calculated in different atmospheric models for a LOS with $\mu=0.1$, taking into account PRD effects both under the AA approximation (solid lines) and in the general AD case (dashed lines). No magnetic field is considered. The considered atmospheric models are: FAL-C (red/orange lines), FAL-A (blue/light blue lines), FAL-F (green/pale green lines), and FAL-P (purple/violet lines). The reference direction for positive $Q$ is taken parallel to the limb.

the quiet Sun (FAL-C), a bright network region of the quiet Sun (FAL-F), and a typical plage area (FAL-P).

Figure 1 shows the emergent $Q / I$ profiles for the aforementioned atmospheric models and a LOS with $\mu=0.1$. In the absence of magnetic fields, the $U / I$ profiles vanish and are consequently not shown. An interesting result is the following: the small trough in the line-core peak of the $Q / I$ profile, obtained when the AA approximation is considered, completely disappears when a general $\mathrm{AD}$ calculation is carried out. The trough found in the AA case can be thus attributed to the averaging over the scattering angle explained in Section 2.4. This clearly highlights the scientific value of $\mathrm{AD}$ calculations, as the sharper $Q / I$ peak attained in this case is in much better agreement with the observations (e.g., Gandorfer 2002). By contrast, a good agreement between AA and AD calculations is found in the wings of the $Q / I$ profile.

$\mathrm{AA}$ and $\mathrm{AD}$ calculations are now compared considering the FAL-C atmospheric model in the absence of magnetic fields for different LOS. The results shown in Figure 2 are consistent with those of Figure 1. For all the considered LOS, AA calculations yield a trough in the line-core peak of the $Q / I$ profile, whereas the AD counterparts show a peak. Additionally, AA and AD calculations substantially agree in the wings of the $Q / I$ profile. As expected, the amplitude of the $Q / I$ profile, both in the core and in the wings, decreases when moving towards the disk center (i.e., for increasing $\mu$ values).

\subsection{Magnetic case}

The linear scattering polarization signal of the Ca I $4227 \AA$ line is primarily sensitive to the presence of magnetic fields through the combined action of the Hanle and magneto-optical effects. The Hanle effect operates in the line core, causing a reduction of the degree of linear polarization and a rotation of the plane of linear polarization. The Hanle critical field for this line is of

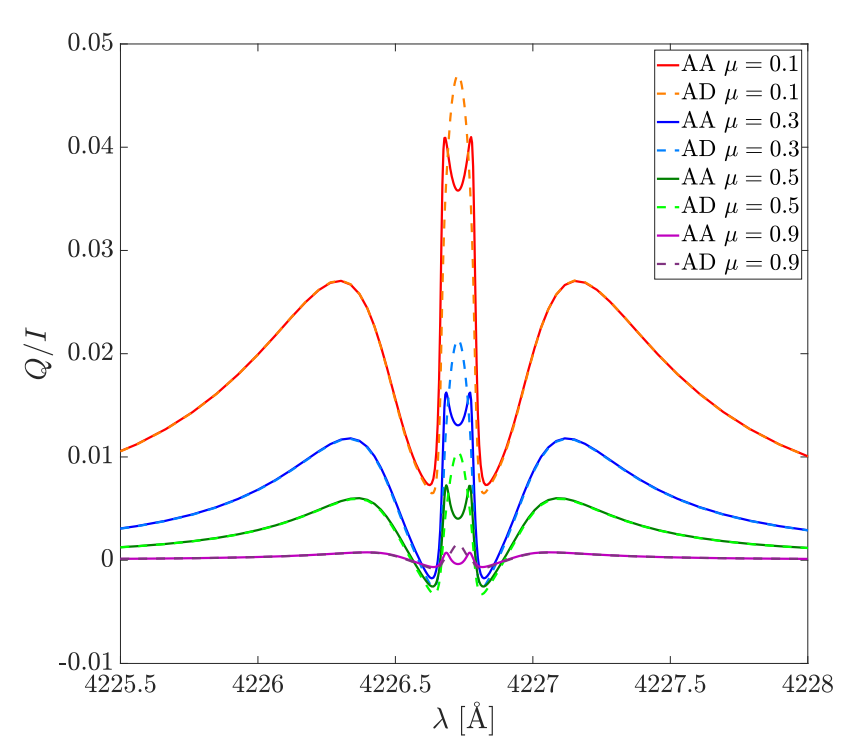

Fig. 2. Emergent $Q / I$ profiles calculated in the FAL-C atmospheric model for different LOS, taking into account PRD effects both under the AA approximation (solid lines) and in the general AD case (dashed lines). No magnetic field is considered. The considered LOS are: $\mu=0.1$ (red/orange lines), $\mu=0.3$ (blue/light blue lines), $\mu=0.5$ (green/pale green lines), and $\mu=0.9$ (purple/violet lines). The reference direction for positive $Q$ is taken parallel to the limb.

approximately $25 \mathrm{G}$. Magneto-optical effects mainly operate in the line wings, producing a rotation of the plane of linear polarization, and a reduction of the linear polarization degree. The impact of this mechanism becomes appreciable in the presence of longitudinal magnetic fields with strengths similar to those required for the Hanle effect to have a substantial impact (more details in Alsina Ballester et al. 2018, 2019). The polarization of the Ca I $4227 \AA$ line is also sensitive to the well-known Zeeman effect, which produces measurable circular polarization signals in the presence of magnetic fields with strengths larger than $10 \mathrm{G}$ in the upper solar photosphere.

Figure 3 shows the impact of the AD treatment on the emergent Stokes profiles of the Ca I $4227 \AA$ line, for a LOS with $\mu=0.1$, when including magnetic fields. The direction of the magnetic field is horizontal and contained in the plane defined by the local vertical and the LOS, while different field strengths have been considered. For the considered range of magnetic field strengths (between 0 and $50 \mathrm{G}$ ), the Hanle effect produces a decrease of the amplitude of the line-core peak of the $Q / I$ profile (till the disappearance of the peak), while giving rise to a peak in the core of the $U / I$ profile. A trough is found in both peaks when performing AA calculations, while the $\mathrm{AD}$ counterparts show a peak. The same figure allows appreciating the sensitivity of the line wings to the same magnetic fields through magneto-optical effects: as the magnetic field increases, the wing lobes of $Q / I$ decrease, while similar wing lobes appear in $U / I$. At such wing wavelengths, AA and AD calculations show a good agreement, thus suggesting that the AA approximation could be suitable to model and exploit magneto-optical effects in the scattering polarization wings of the Ca I $4227 \AA$ line.

\section{Conclusions}

This paper presents a quantitative comparison between the AA and AD approaches in the numerical solution of the NLTE RT 

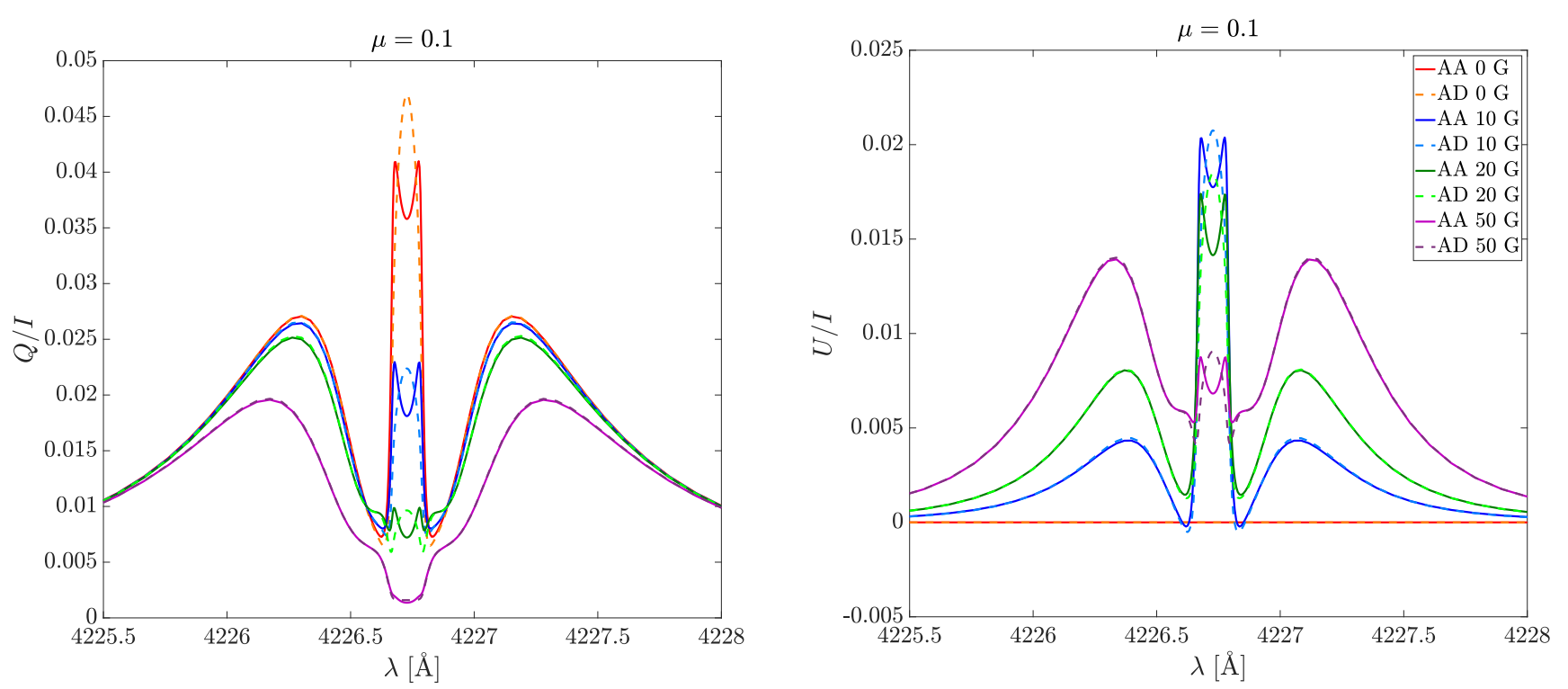

Fig. 3. Emergent $Q / I$ (left panel) and $U / I$ (right panel) calculated in the FAL-C atmospheric model for a LOS with $\mu=0.1$, taking into account PRD effects both under the AA approximation (solid lines) and in the general AD case (dashed lines). The magnetic field is horizontal and contained in the plane defined by the local vertical and the LOS. Different field strengths have been applied: $0 \mathrm{G}$ (red/orange lines), $10 \mathrm{G}$ (blue/light blue line), $20 \mathrm{G}$ (green/pale green lines), and $50 \mathrm{G}$ (purple/violet lines). The reference direction for positive $Q$ is taken parallel to the limb.

problem of polarized radiation, taking scattering polarization and PRD effects into account. In particular, the $Q / I$ and $U / I$ Stokes profiles of the Ca r $4227 \AA$ spectral line have been modeled and discussed, considering realistic $1 \mathrm{D}$ semi-empirical atmospheric models in the presence of magnetic fields.

The most relevant feature of the polarization signals obtained when performing AD calculations is the disappearance of the artificial trough in the line-core peak of the $Q / I$ and $U / I$ scattering polarization profiles obtained when using the AA approximation. This difference between AA and AD calculations is found for all LOS, both in the absence and in the presence of magnetic fields. This finding highlights the scientific value of AD calculations, as the corresponding line-core peak (which is a key observable for Hanle effect diagnostics) is in much better agreement with the observations. Previous investigations have shown that other strong resonance lines, such as $\mathrm{Na}$ I $\mathrm{D}_{2}$, show a trough in the line core peak of the $Q / I$ profile when modeled under the AA approximation (e.g., Belluzzi et al. 2015; Alsina Ballester et al. 2021). It is expected that also in these cases the trough will disappear when AD calculations are carried out.

Additionally, the numerical results support the validity of the AA approximation for modeling the wings of the $Q / I$ and $U / I$ profiles in the static case. This validates the investigation on the Ca I $4227 \AA$ line performed by Alsina Ballester et al. (2018) under the AA approximation, strengthening the diagnostic potential of magneto-optical effects for exploring the magnetism of stellar atmospheres.

The numerical approach presented in this paper can be applied to model other resonance lines, and can be generalized to a wider range of scenarios, such as two-term atomic models, and 3D atmospheric models with arbitrary magnetic and bulk velocity fields. In particular, the impact of bulk velocity motions on the modeling of the scattering polarization of resonance lines taking PRD effects into account in the general AD case will be subject to an upcoming investigation.
Acknowledgements. The financial support by the Swiss National Science Foundation (SNSF) through grant CRSII5_180238 is gratefully acknowledged. E.A.B. and L.B. gratefully acknowledge financial support by SNSF through grant 200021_175997. J.T.B. acknowledges the funding received from the European Research Counsil (ERC) under the European Union's Horizon 2020 research and innovation programme (ERC Advanced Grant agreement No $742265)$.

\section{References}

Alsina Ballester, E., Belluzzi, L., \& Trujillo Bueno, J. 2016, ApJ, 831, L15 Alsina Ballester, E., Belluzzi, L., \& Trujillo Bueno, J. 2017, ApJ, 836, 6 Alsina Ballester, E., Belluzzi, L., \& Trujillo Bueno, J. 2018, ApJ, 854, 150 Alsina Ballester, E., Belluzzi, L., \& Trujillo Bueno, J. 2019, ApJ, 880, 85 Alsina Ballester, E., Belluzzi, L., \& Trujillo Bueno, J. 2021, Phys. Rev. Lett., accepted

Anusha, L. S., Nagendra, K. N., Bianda, M., et al. 2011, ApJ, 737, 95

Belluzzi, L. \& Trujillo Bueno, J. 2012, ApJ, 750, L11

Belluzzi, L. \& Trujillo Bueno, J. 2014, A\&A, 564, A16

Belluzzi, L., Trujillo Bueno, J., \& Landi Degl'Innocenti, E. 2015, ApJ, 814, 116 Belluzzi, L., Trujillo Bueno, J., \& Štěpán, J. 2012, ApJ, 755, L2

Benedusi, P., Janett, G., Belluzzi, L., \& Krause, R. 2021, A\&A, accepted Bianda, M., Ramelli, R., Anusha, L. S., et al. 2011, A\&A, 530, L13

Bianda, M., Stenflo, J. O., Gandorfer, A., \& Gisler, D. 2003, in Astronomical Society of the Pacific Conference Series, Vol. 286, Current Theoretical Models and Future High Resolution Solar Observations: Preparing for ATST, ed. A. A. Pevtsov \& H. Uitenbroek, 61

Bommier, V. 1997a, A\&A, 328, 706

Bommier, V. 1997b, A\&A, 328, 726

Capozzi, E., Ballester, E. A., Belluzzi, L., et al. 2020, A\&A, 641, A63

Carlin, E. S. \& Bianda, M. 2017, ApJ, 843, 64

Casini, R., del Pino Alemán, T., \& Manso Sainz, R. 2017, ApJ, 848, 99

Casini, R., Landi Degl'Innocenti, M., Manso Sainz, R., Land i Degl'Innocenti, E., \& Landolfi, M. 2014, ApJ, 791, 94

Collados, M., Lagg, A., Díaz Garcí A, J. J., et al. 2007, in ASP Conf. Ser., Vol. 368, The Physics of Chromospheric Plasmas, ed. P. Heinzel, I. Dorotovič, \& R. J. Rutten, 611

del Pino Alemán, T., Trujillo Bueno, J., Casini, R., \& Manso Sainz, R. 2020, ApJ, 891, 91

Dumont, S., Omont, A., Pecker, J. C., \& Rees, D. 1977, A\&A, 54, 675

Faurobert, M. 1987, A\&A, 178, 269

Faurobert, M. 1988, A\&A, 194, 268

Faurobert-Scholl, M. 1992, A\&A, 258, 521

Fontenla, J. M., Avrett, E. H., \& Loeser, R. 1993, ApJ, 406, 319 
Gandorfer, A. 2000, The Second Solar Spectrum: Volume I (Hochschulverlag AG, ETHZ)

Gandorfer, A. 2002, The Second Solar Spectrum: Volume II (Hochschulverlag AG, ETHZ)

Gray, D. F. 1992, Observation and Analysis of Stellar Photospheres (Cambridge: Cambridge University Press)

Hackbusch, W. 1994, Applied Mathematical Sciences, Vol. 95, Iterative Solution of Large Sparse Systems of Equations, 1st edn. (New York: Springer)

Ishikawa, R., Bueno, J. T., del Pino Alemán, T., et al. 2021, Science Advances, 7, eabe8406

Janett, G., Benedusi, P., Belluzzi, L., \& Krause, R. 2021, A\&A, accepted

Janett, G., Carlin, E. S., Steiner, O., \& Belluzzi, L. 2017, ApJ, 840, 107

Janett, G. \& Paganini, A. 2018, ApJ, 857, 91

Janett, G., Steiner, O., \& Belluzzi, L. 2018, ApJ, 865, 16

Jaume Bestard, J., Trujillo Bueno, J., Štěpán, J., \& del Pino Alemán, T. 2021, ApJ, 909, 183

Kano, R., Trujillo Bueno, J., Winebarger, A., et al. 2017, ApJ, 839, L10

Landi Degl'Innocenti, E. \& Landolfi, M. 2004, Astrophysics and Space Science Library, Vol. 307, Polarization in Spectral Lines (Dordrecht: Kluwer Academic Publishers)

Leenaarts, J., Pereira, T., \& Uitenbroek, H. 2012, A\&A, 543, A109

Mihalas, D. 1978, Stellar Atmospheres, 2nd edn. (San Francisco: W.H. Freeman and Company)

Nagendra, K. N., Frisch, H., \& Faurobert, M. 2002, A\&A, 395, 305

Ramelli, R., Balemi, S., Bianda, M., et al. 2010, in Society of Photo-Optical Instrumentation Engineers (SPIE) Conference Series, Vol. 7735, Proc. SPIE, $77351 Y$

Rees, D. E. \& Saliba, G. J. 1982, A\&A, 115,

Sampoorna, M. \& Nagendra, K. N. 2015, ApJ, 812, 28

Sampoorna, M., Nagendra, K. N., \& Frisch, H. 2011, A\&A, 527, A89

Sampoorna, M., Nagendra, K. N., \& Stenflo, J. O. 2008, ApJ, 679, 889

Sampoorna, M., Nagendra, K. N., \& Stenflo, J. O. 2017, ApJ, 844, 97

Sampoorna, M., Trujillo Bueno, J., \& Landi Degl'Innocenti, E. 2010, ApJ, 722, 1269

Seaton, M. J. 1962, Proceedings of the Physical Society, 79, 1105

Stenflo, J. 1994, Solar Magnetic Fields: Polarized Radiation Diagnostics, Vol. 189 (Springer)

Stenflo, J. O. 1982, Sol. Phys., 80, 209

Stenflo, J. O., Baur, T. G., \& Elmore, D. F. 1980, A\&A, 84, 60

Sukhorukov, A. V. \& Leenaarts, J. 2017, A\&A, 597, A46

Supriya, H. D., Nagendra, K. N., Sampoorna, M., \& Ravindra, B. 2012, MNRAS, 425,527

Supriya, H. D., Sampoorna, M., Nagendra, K. N., Ravindra, B., \& Anusha, L. S. 2013, J. Quant. Spectr. Rad. Transf., 119, 67

Supriya, H. D., Smitha, H. N., Nagendra, K. N., et al. 2014, ApJ, 793, 42

Traving, G. 1960, Über die Theorie der Druckverbreiterung von Spektrallinien (Karlsruhe: Braun)

Trujillo Bueno, J. 2001, in Astronomical Society of the Pacific Conference Series, Vol. 236, Advanced Solar Polarimetry - Theory, Observation, and Instrumentation, ed. M. Sigwarth, 161

Trujillo Bueno, J., Landi Degl'Innocenti, E., \& Belluzzi, L. 2017, Space Sci. Rev., 210, 183

Trujillo Bueno, J., Štěpán, J., Belluzzi, L., et al. 2018, ApJ, 866, L15

Uitenbroek, H. 2001, ApJ, 557, 389

Unsöld, A. 1955, Physik der Sternatmospharen, mit besonderer Berücksichtigung der Sonne. (Berlin: Springer)

Štěpán, J. 2015, in IAU Symposium, Vol. 305, Polarimetry, ed. K. N. Nagendra, S. Bagnulo, R. Centeno, \& M. Jesús Martínez González, 360-367 